\title{
An Intelligent Decision-Support System for Air Cargo Palletizing
}

\author{
No-San Lee \\ University of Cologne \\ lee@wim.uni-koeln.de
}

\author{
Philipp Gabriel Mazur \\ University of Cologne \\ mazur@wim.uni-koeln.de
}

\author{
Moritz Bittner \\ University of Cologne \\ bittner@wim.uni-koeln.de
}

\author{
Detlef Schoder \\ University of Cologne \\ schoder@wim.uni-koeln.de
}

\begin{abstract}
Palletizing in the air cargo sector faces a large number of constraints (e.g., aviation safety regulations) and represents a highly complex problem. In air cargo operations, there is hardly any digital support to optimize the palletizing process. As a result, desired objectives (e.g., optimal utilization of the possible loading weight, maximum use of the available loading space, or both) are often only met by chance. The goal of this research is to report on the design and performance of an intelligent decision support system that we built for the air cargo context. This system supports the manual palletizing process by considering far more constraints as well as more complex item shapes and unit load devices than any other system we know. We explain the problem context, including the essential requirements; model the solution design; and develop the intelligent decision support system as an artifact, which we then evaluate.
\end{abstract}

\section{Introduction}

Air cargo transportation surpasses other modes of transportation in terms of delivery speed and reliability [20] and has thus become increasingly popular. Indeed, between 2014 and 2019, the air cargo market grew steadily, and cargo volumes increased by about $20 \%$ [16]. At the same time, global air traffic was responsible for about 918 million tons of $\mathrm{CO} 2$ in 2018, which is about $2.4 \%$ of global $\mathrm{CO} 2$ emissions from fossil fuels, thus putting pressure on the industry to reduce costs and optimize the use and consumption of resources [11]. On the other hand, only $1 \%$ of the world's cargo is transported by air, but this portion accounts for about $35 \%$ of overall cargo value [1]. As such, better utilization of the available transport volume can have a significant and positive impact on economic returns.

Ground handling agents recognize that digital transformation offers a solution to meet the existing cost, time, and performance pressures in the air cargo sector. These systems are already used to support and optimize airline processes and systems, including passenger and baggage handling, lounge services, and staff planning and scheduling [17].

However, when it comes to palletizing air cargo, there is hardly any digital support for optimizing the palletizing process and reducing the loading time of cargo on so-called unit load devices (ULDs). ULDs are standardized and heterogeneous pallets or containers on or in which cargo is positioned. A human palletizer places individual cargo items onto or into a ULD either by hand or by forklift. As such, the quality of a palletizing solution strongly depends on the experience and creativity of the palletizer. When the palletizing process becomes more complex, inexperienced palletizers usually follow their own heuristics in combination with a trial-and-error approach. In doing so, desired goals (e.g., optimal utilization of the possible load combined with maximum utilization of the available loading space within a narrow time window) are often only achieved by chance. Only through years of professional experience is it possible for individuals to continuously improve their palletizing skills to achieve the desired results satisfactorily. On the other hand, there is a clear shortage of qualified personnel in air cargo operations and the risk of a loss of knowhow due to the age-related retirement of air cargo employees in the near future [6].

In research, especially in operations research, this NP-hard problem is called the pallet loading problem (PLP) or container loading problem (CLP) [4, 5]. Research in this area usually seeks solutions using exact methods or heuristics [27]. The resulting research artifacts are well understood theoretically, but to cope with complexity, they often ignore constraints from reality as well as non-cuboid and irregular cargo items. In particular, these constraints often relate to the process of pallet and container loading. Specific constraints [5, 24] and/or object shapes [10] may also arise depending on industrial characteristics. These constraints are discussed in the literature [3,4] but are not fully applied in research approaches. One reason for the lack of practical relevance of current PLP and CLP approaches, especially in the air cargo sector, is 
that research does not consider all relevant constraints $[4,24]$, and the feasibility of solution approaches is usually demonstrated by testbeds with cube-shaped or cuboid objects only $[3,9]$. In the air cargo context, the complexity of the overall problem is greatly increased due to the complex shapes of many cargo items and ULDs as well as the constraints imposed by strict aviation safety regulations. Current solutions in research and practice do not reflect this full complexity. As a result, reality is not adequately reflected, so there is a lack of practical and feasible solutions.

For this reason, the goal of this research is to report on the design and performance of an intelligent decision support system (IDSS) we built for the air cargo context. This system supports the manual palletizing process by considering far more constraints as well as more complex item and ULD shapes than any other system we know. The IDSS generates practicable solutions via an applied genetic algorithm (GA) and supports human palletizers before and during physical assembly by visualizing, monitoring, and validating the generated palletizing solutions.

After presenting related work, we narrow down the problem context and describe the associated requirements that the solution design must consider (e.g., by enumerating all relevant constraints based on input from a large air cargo company). Furthermore, we present the solution design and the results of the evaluation of the IDSS followed by a discussion and conclusion of this research work.

\section{Related Work}

The PLP belongs to the family of threedimensional cutting and packing problems, in which a set of small items must be grouped and assigned to a set of larger items [26]. A distinction can be made between the manufacturer's problem (MP) and the distributor's problem (DP) [13]. In the MP, homogenous items (i.e., identical boxes) are placed onto identical pallets. In the DP, heterogenous items of varying dimensions are placed onto standardized pallets. Due to its non-repetitive nature and solution time requirements, the DP is more difficult [13].

The PLP is closely related to the CLP, and many approaches can be assigned to the same problem classifications [2]. One of the few differences between these problems is that research on CLP assumes that the primary object is a rigid-walled container that supports cargo laterally. Such rigid walls are usually replaced by a safety net on pallets [2].

In the literature, numerous studies have explored the mentioned loading problems. A comprehensive overview of the CLP is provided by Bortfeldt and
Wäscher [4]. They review the literature with regard to practical constraints based on Bischoff and Ratcliffe's work [3] and conclude that only very few papers consider multiple real-world constraints simultaneously. Pollaris et al. [24] update this review by focusing on the vehicle routing problem and come to a similar conclusion regarding the simultaneous inclusion of practical constraints in loading solutions. Zhao et al. [27] provide a complementary review to Bortfeldt and Wäscher [4] focusing on the design and implementation of solution methods for the CLP. Exact methods (e.g., mathematical models or mixed integer programming [MIP]); placement heuristics (e.g., layer or wall-building approaches); and improvement heuristics or metaheuristics, such as tabu search and genetic algorithm are presented as common solution methods [27].

Although these previous studies provide useful insights, there is little research on air cargo, which has its own specifics. Only MIP approaches are found in the air cargo context $[14,22]$, sometimes in combination with placement heuristics [8] or metaheuristics [21]. All approaches consider the heterogeneous shapes of ULDs [8, 21, 22], with the exception of Hong Ha and Nananukul [14], who use a cuboid ULD container for their MIP model. The recent paper by Brandt and Nickel [5] offers a detailed literature review focusing on the air cargo context, which also contains the papers mentioned before. Their research provides a consolidated problem definition of air cargo loading planning and subordinates the loading problem of ULDs as a subproblem. Furthermore, they illustrate that in the air cargo context, research on loading problems does not consider all real-world constraints simultaneously, in line with Bortfeldt and Wäscher [4] and Pollaris et al. [24].

In addition to the constraints, consideration of strongly heterogeneous, non-cuboid or irregular items is necessary to realistically address the loading problems in the air cargo context. Only a few studies have examined irregular placement problems for the two-dimensional case, while research on the threedimensional case is even more scarce and primarily focuses on item placement. One of the very few examples is the paper by Egeblad et al. [10], which investigates container loading of irregular shapes from the perspective of a furniture manufacturer. The authors divide the input items into the categories of large (mainly irregular items), medium (boxes), and small (boxes) and employ multiple heuristics for each shape type.

\section{Methodology}


To achieve our research goal, we applied a designoriented approach following a design science research (DSR) methodology [23]. Prior to designing and developing our solution, we determined which quantitative and qualitative criteria must be met to capture and narrow down the problem context in order to solve the defined problem. In cooperation with a large air cargo company, we conducted joint workshops with experts, observed operations onsite at the cargo hub, and conducted consecutive interviews with palletizers over a period of several months. We also conducted a literature review, which allowed us to draw on existing models and approaches when designing our solution, including optimization approaches for loading problems in both operations research and generative design. The definition of the problem context follows the reasoning in DSR that "making improvements should . . . be formally grounded in kernel theories from the knowledge base" [12] based on previous development methods and theoretical results.

Based on the defined problem context, which includes the essential requirements, we modeled the solution design, developed the IDSS as an artifact, and then evaluated the IDSS. These steps correspond to the DSR approach of proving the feasibility of a problem solution and then evaluating its performance. In the following, we present the definition of the problem context, the solution design, and the results of our evaluation.

\section{Problem Context: Air Cargo Palletizing}

\subsection{Size and Shape Heterogeneity}

Air cargo has an enormous variety of cargo items and pallet contours, as shown in Figure 1. Therefore, palletizing problems in the air cargo context can be classified as DPs [13]. Among other things, cargo items differ in shape, packaging material, weight, load capacity, and substructure. Most items have a cuboid shape and are often delivered pre-palletized on a wooden pallet or grouped together to form one multipiece item. Irregular item shapes are less common [5] but require higher loading effort as they cannot simply be placed on a pallet. In particular, irregular items cannot be placed haphazardly because they must be arranged on a pallet such that the final palletizing layout corresponds to a given pallet contour (although pallets and contours may vary in type and size). For containers with solid metal walls, the contour is already fixed and rigid.

To the best of our knowledge, no statistics currently exist regarding the frequency of different cargo item sizes and shapes. According to the results of our workshops, experts estimate that about $95 \%$ of objects are cuboid in shape, but they are strongly heterogeneous in terms of their dimensions. The remaining 5\% of non-cuboid and irregular items have unpredictable variance in size and shape, which greatly increases the complexity of palletizing.

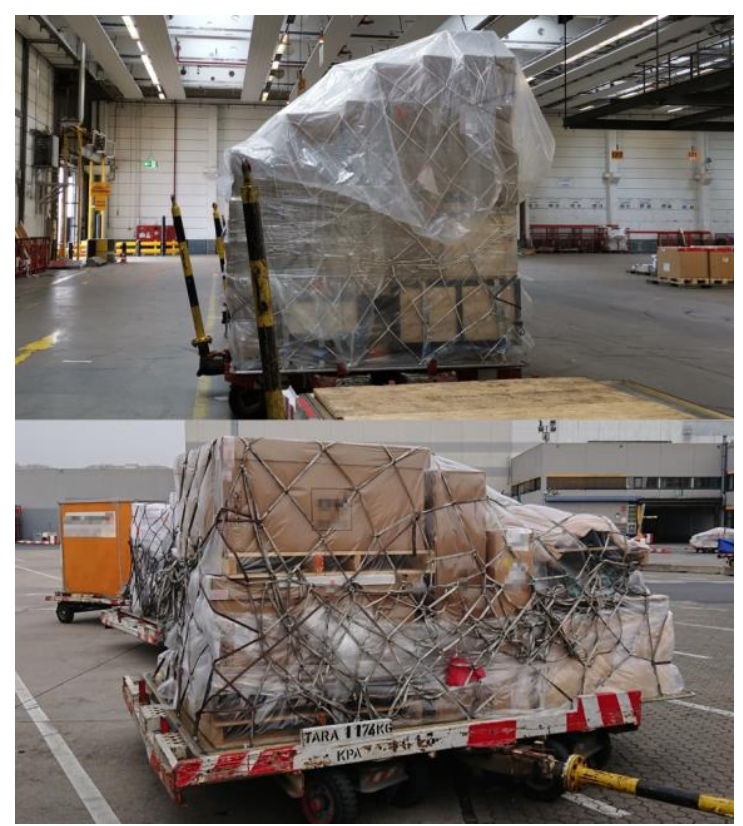

Figure 1. Heterogeneous cargo items and pallet contours

According to the experts we interviewed, this unpredictable variance has a direct influence on palletizing efficiency. For example, an increasing heterogeneity of cargo items has a negative influence on the load factor of a load device. The load factor is one of the most important measurements in palletizing and represents loading efficiency in terms of the load volume used. The load factor is calculated by relating the load volume used by already loaded cargo items to the maximum available load volume of the load device when empty. According to Brandt and Nickel [5], the physical volume capacity of aircrafts could only be utilized to 60 or $70 \%$ for most flights. If we break this down to the ULD level, the average utilization of a loaded ULD should be also within these percentage ranges. The experts noted that a loaded container with a load factor of $75 \%$ and more is desirable. Furthermore, from a load factor of $75 \%$, no additional load securing of the cargo in the container by nets or tie-down straps is required. However, a load factor of $75 \%$ or above is a threshold that experienced palletizers can typically reach. Indeed, achieving a good load factor depends on other factors other than item heterogeneity, such as the weight and size of the cargo or regulatory requirements, which may prevent 
the desired load factor from being achieved. Nevertheless, as a key figure, the load factor of an ULD generally provides a significant way of estimating loading efficiency.

\subsection{Constraints in Air Cargo Palletizing}

When air cargo pallets are loaded, major constraints must be considered. A distinction is made between hard and soft constraints [4]. Hard constraints must be fulfilled to find a palletizing solution. In contrast, soft constraints tolerate deviations within defined limits. One such constraint is the time window within which a pallet must be completed. In addition, there are a number of strict aviation safety regulations, which are primarily standardized by the International Air Transport Association (IATA) through its Cargo Handling Manual [15].

In summary, there are essentially six aviation safety-related (ASR) constraints that a pallet must meet to be transported by air. They are all hard constraints, with the exception of the balance, which needs to be within a given tolerance range. These constraints, which we briefly describe in the following, primarily relate to more complex pallet loading but can also be applied to containers.

Stability (ASR1): A distinction is made between static and dynamic stability. A pallet is statically stable if it is able to withstand gravity at every stage of its construction. Dynamic stability describes a pallet's ability to withstand the centrifugal forces that may occur during transport.

Maximum Weight (ASR2): The total weight of cargo items on the pallet must not exceed a certain weight limit. The actual value of the weight limit depends on the type of pallet used, the regulatory requirements, and the intended position of the pallet in the aircraft.

Floor Load (ASR3): The floor load describes a fixed maximum point load per square meter that must not be exceeded during palletizing. If this limit is exceeded, the pallet may become physically deformed, making it difficult or practically impossible to secure the load in the aircraft.

Balance (ASR4): The weight of the cargo items should be evenly distributed across the base area of the pallet. This facilitates pallet transportation, and in the case of air cargo, it may influence the balancing of the airplane.

Incompatibility (ASR5): This constraint combines the characteristics of separation and positioning constraints. Due to regulatory requirements, especially for certain types of goods (e.g., dangerous goods, live animals, etc.), it is necessary to load these goods either separately on different pallets or at a certain distance on the same pallet.

Contour (ASR6): The cargo items must be placed within the pallet contour. While certain oversized cargo is allowed to overhang and extend over two or more pallets, this is a special case that goes beyond our consideration.

Overall, an optimized palletizing solution can only be achieved by considering the aforementioned ASR constraints as well as other known constraints discussed in the literature $[4,5,24]$. Therefore, for the practicable realization of palletizing solutions, it is essential to consider additional constraints for palettizing (ACPs), which we derived from our workshops and interviews and then mapped with literature.

Load Priority (ACP1): Higher-priority items should be preferred or fully loaded on a pallet before lower-priority items $[4,5,24]$. This constraint can be set as either hard or soft depending on the priority of the items that must be loaded.

Stacking (ACP2): The way items are stacked may be limited to protect against damage [4, 5, 24]. For example, some items may have limits regarding how much weight can rest on them. This usually hard constraint also reflects the fragility of a cargo item.

Item Grouping (ACP3): This soft constraint is related to which items are to be loaded together on the same pallet, for example, to meet customer demands, to combine partial deliveries from the same consignor, or to group items with identical transport destinations $[4,5,24]$.

Non-overlap (ACP4): The items on the pallet must not overlap [4, 24]. Even though this hard constraint is not physically possible, it must be considered in the solution finding process by computer programs and algorithms.

Orthogonality (ACP5): Rectangular objects like cube-shaped or cuboid items must be placed on the palette so that the edges of the objects are loaded orthogonal or parallel to the rectangular edges of the palette or contour [24]. This hard constraint serves to facilitate system development. However, it has a direct influence on the physical loading of a pallet and makes it easier for the palletizer to handle rectangular shaped items more efficiently in reality.

Item Orientation (ACP6): The items may only be rotated in certain directions [4, 5, 24]. This constraint may be due to hard loading restrictions. For example, cargo items that are already pre-palletized on a wooden pallet can only be rotated vertically and not upside down.

Complexity (ACP7): A palletizing solution must be realizable and executable by a human palletizer, a forklift driver, or even a palletizing robot depending 
on the pre-defined problem context, size and weight of the cargo, and the available machines [4]. Above all, this hard constraint concerns the loading sequence of the cargo and from which loading direction the pallet has to be loaded. For containers, possible loading directions are specified by doors or openings. In the case of manual loading without mechanical support, the maximum permissible load of the human palletizer must be considered for occupational health and safety reasons.

Positioning (ACP8): This hard constraint relates to absolute or relative positioning [4, 5, 24]. For absolute positioning, items must be loaded to certain positions on the pallet, for example, to reach them more easily. Relative positioning determines whether a minimum distance between items must be maintained when items are loaded together (see also incompatibility).

Separation (ACP9): This hard constraint relates to separating items that must not be loaded together on a single pallet $[4,24]$. This separation may be needed due to regulatory requirements or to the conflicting nature of the goods, which excludes loading them together on the same pallet (see also incompatibility).

Complete Shipment (ACP10): Groups of cargo items may be included entirely or not at all [4, 5, 24]. This hard constraint applies especially to cargo items that may only be transported as a whole, such as several individual parts belonging to a single car or engine.

\section{Solution Design}

\subsection{Genetic Algorithms}

The developed IDSS calculates a practicable solution for palletizing by applying a GA as a metaheuristic approach. GAs have become well established in research on three-dimensional cutting and packaging problems with high complexity and several optimization goals [7, 27]. In our case, using a GA is a suitable approach due to the high heterogeneity of items and ULDs in terms of size and shape. Therefore, each new combination of item sets and ULDs always represents a new palletizing task and thus always requires a new instantiation of optimization by the GA. This approach goes along with the non-repetitive nature of palletizing problems in the air cargo context according to their classification as DPs. Furthermore, a GA is preferable to exact methods. Unlike many other exact methods, GAs use stochastic operators instead of deterministic operators and have the ability to move freely through the solution space without context information such that the degree of adaptation of an individual solution is only evaluated with the help of a fitness function [18].

For our research, we use a basic GA approach following Kramer [18]. The algorithm is shown as pseudo code in Figure 2. It starts with an initial set of arbitrary solution candidates and recombines and mutates them to generate new solutions. The underlying assumption is that the new solutions may be better than the old solutions. The solutions' fitness is then assessed using self-defined fitness criteria, and the worst solutions are eliminated. The remaining superior solutions are then recombined and mutated again. This process is repeated until satisfying solutions are found.

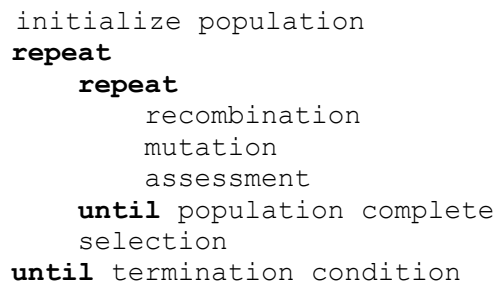

Figure 2. Applied GA in pseudo code following Kramer [18]

In our case, we modelled the identified constraints from Section 4.2. as individual fitness criteria, which were then weighted and combined linearly to form an overall fitness score. The constraints vary in their degree of satisfaction from simply modelled geometric and mechanical relationships (e.g., balance, contour) to a simulation with a real-time physics engine (e.g., stability). The applied GA enhances its solutionfinding capabilities with higher iteration rates and greater population sizes due to the resulting higher coverage of the solution space. However, the enhancement of the solution-finding capabilities is at the expense of the runtime for the calculation of the solutions, which is increased by this.

\subsection{User Interactions with the IDSS}

The main users of the IDSS are the supervisor, who is responsible for monitoring and validating the palletizing solutions, and the palletizer, who performs the physical assembly. Despite the automation made possible by the GA approach, the supervisor must approve finished ULDs before they can be loaded onto an aircraft [15]. There is also the question of how users can modify the solutions generated by the GA due to changing conditions and environmental factors. As such, the system design must consider the human element and should be able to combine human intelligence and machine intelligence to search for 
satisfactory solutions and to adapt the implemented algorithm. Specifically, there are three types of user interactions with the IDSS that affect the generation of the palletizing layout solution: (1) the possibility to recalculate palletizing solutions if the user is not satisfied with them, (2) the possibility to modify the configuration of the GA to improve the solutionfinding capabilities of the algorithm if necessary, and (3) the possibility to modify the configuration of the assessment functionalities if the tolerance ranges of the constraints have to be altered.

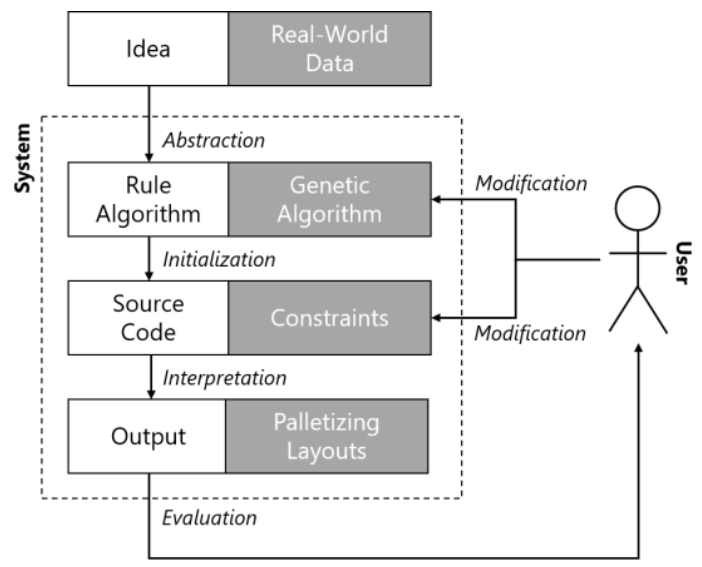

\section{Figure 3. Adapted GDS process flow following Krish [19]}

In the first case, a user interface (UI) must be provided that allows users to validate the generated palletizing layouts and trigger a recalculation if they deem a layout to be unsatisfactory. For the challenges resulting from the second and third interaction types, the field of generative design systems (GDSs) offers a solution approach that explicitly includes user interactions to modify algorithms and constraints.

The primary goal of a GDS is to use "computational capabilities to support human designers and (or) automate parts of the design process" [25]. In this context, palletizing solutions can be regarded as layout patterns or design layouts because the palletizing process in the air cargo context can be seen as a non-repetitive, highly complex, and creative design process that relies heavily on prior knowledge, experience, and creative solution competence. In a GDS-supported design process, the user plays a central role by continuously modifying the generative schema upon which the end results are based, with which the solution space is traversed in search of practicable design solutions [19]. IDSS users also need to undertake these necessary tasks to adjust and modify the configuration of the GA and the constraints. This area of responsibility is completely new in the air cargo sector and does not even exist at present. Nevertheless, these necessary user interactions must be considered in our system design to allow modifications to the configurations of the algorithm and assessment functionalities. For this purpose, a GDS approach provides a way to implement our IDSS through a cooperative constraintbased human-machine interaction system design [19].

Figure 3 shows the GDS process flow following Krish [19], which we adapted to the palletizing support conditions in the air cargo context to generate optimized palletizing layouts. Instead of the typical idea input used at the beginning of a design process, we use applicable real-world data as our input. We use a GA as the rule algorithm. The source code is represented by the constraints, which are implemented as assessment functionalities. Finally, the generated palletizing layouts are the output, which can then be evaluated by the user. The user can also make necessary adjustments to the system by modifying the GA as well as the constraints.

\subsection{System Design}

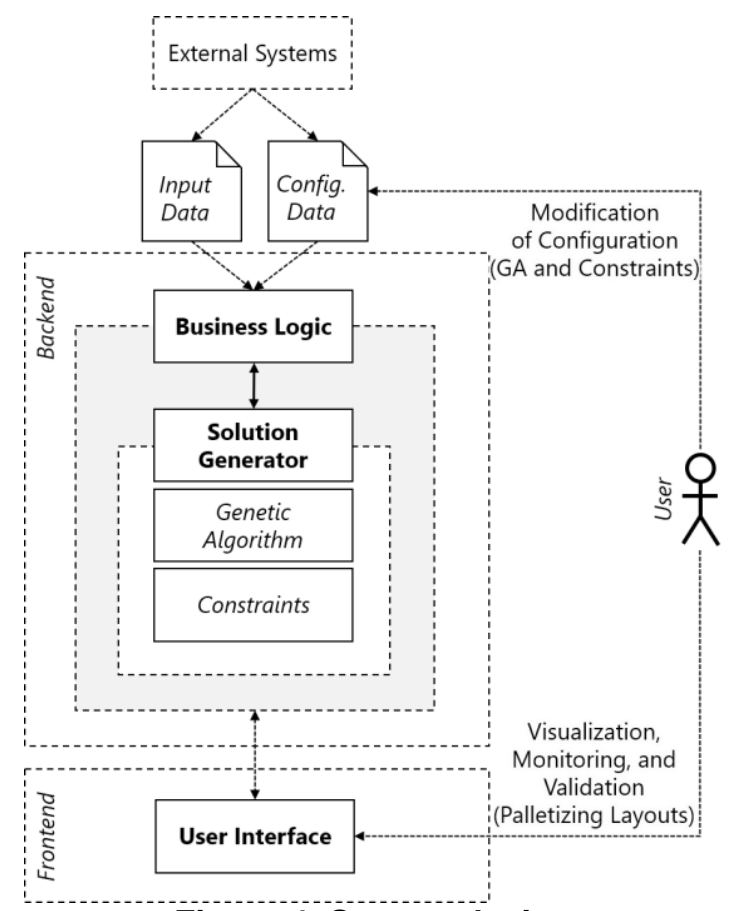

Figure 4. System design

Figure 4 shows the system design of our implemented IDSS, which supports the palletizing process and necessary user interactions at various points. The main system features include the generation of optimized and practicable palletizing layouts; the visualization, monitoring, and validation of the generated palletizing layouts; and support for physical palletizing by the UI. On the architectural 
level, the palletizing layouts are calculated in the backend by the solution generator (SG). The SG is the heart of the system and consists of the GA and the assessment functionalities for the constraints.

The frontend guides the user through the process across multiple screens and is connected to the backend via REST and WebSocket APIs as data interfaces. For this purpose, the backend provides suitable data interfaces for data input and output via a business logic layer. This enables external systems to be connected as data input sources. Input data are necessary for generating palletizing layouts, and configuration data are needed to establish the settings of the SG. The configuration data enable external systems or users to make necessary modifications to the algorithm or the assessment functionalities.

\section{Evaluation}

\subsection{Dataset}

Our evaluation of the IDSS was carried out with special attention to the practical relevance of the palletizing layouts. To the best of our knowledge, no complete test dataset containing sufficient information for operational handling in a practical context is currently available for research purposes. However, to keep the practical relevance in focus, we applied Brandt and Nickel's dataset [5]. The authors compiled this dataset from a large air cargo company. The dataset contains cargo-related information, such as outer dimensions, weight, priority, item groupings, incompatibilities between item characteristics, loading capacity, and orientation restrictions. Since the data were taken from a real application, the complexity prevailing in practice can be approximated to a high degree. These data contrast the testbed data commonly used in the literature (e.g. [3, 9]) as testbeds are artificially created and aim to challenge new methods' ability to solve loading problems. It should be noted that the dataset also has some shortcomings. In particular, there is a lack of meta-information about the specific shapes or silhouettes, underlying wooden pallets, packaging materials, and weight distribution of the cargo items.

Two test scenarios were defined for evaluation. In the first scenario (A), Brandt and Nickel's original data [5] were applied. The dataset contains only cuboid items, and we used the original side lengths and the information provided for each single item. For the second scenario (B), we selected $5 \%$ of all existing items and randomly converted them into irregular object shapes, such as cylinders, L-shapes, or polygonal prisms of the same volume, all of which are supported by the IDSS. Examples of the supported item shapes are shown in Figure 5. This artificially created case, with an irregularity factor of about $5 \%$, reflects the findings from operational experience within a cargo hub. We again used the additional information on the cargo items from the original dataset.
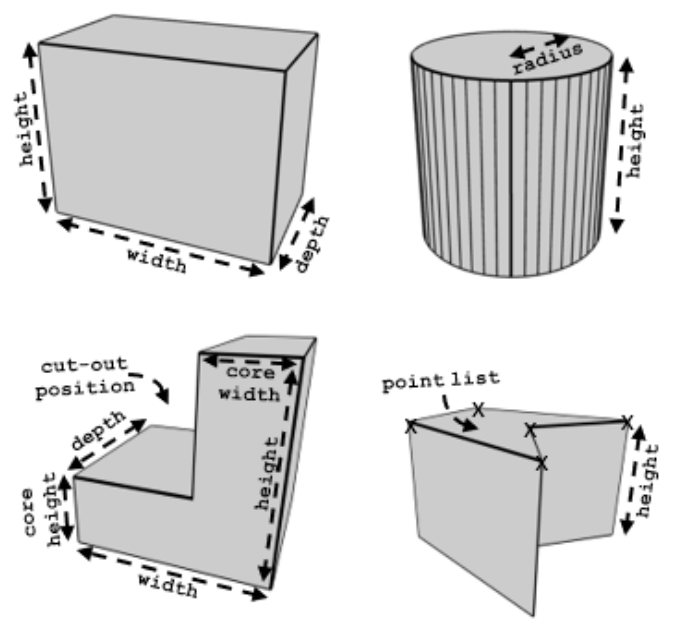

Figure 5. Examples of supported item shapes

For each scenario, we randomly selected five flight segments from the entire dataset and applied them to loading problems for single pallets. We limited the total number of items for a single pallet to 50. These restrictions were set because the SG should load a pallet with only a subset of the cargo for a flight. At the same time, the cumulative volume of the total number of items to be loaded exceeds the available space. This leads to a shortage of the available loading volume of the load device, so the maximum input approach is used. In addition, these restrictions increase the scenarios' realism since items are normally distributed over several load devices in practice.

To underline the suitability of our solution design for problems with heterogeneous pallets and contours, we added three different pallet types with different contours for each scenario. Combining the two scenarios $\mathrm{A}$ and $\mathrm{B}$ and the three pallet types with different contours, we have a total of six different scenario-to-palette/contour combinations. A visual representation of the pallet types and contours included can be found in Figure 6 .

\subsection{Computational Results}

In the following, we present the computational results of our implementation. In addition, a summarized illustration of the measurement outcomes can be found in Table 1 . 
Table 1. Computational results

\begin{tabular}{|c|c|c|c|c|c|c|}
\hline \multirow{2}{*}{$\begin{array}{l}\text { Scenario } \\
\text { Pallet/Contour }\end{array}$} & \multicolumn{3}{|c|}{ A (Original) } & \multicolumn{3}{|c|}{ B (Irregular) } \\
\hline & 1 (PMC/F) & $2(\mathrm{PAG} / \mathrm{Y})$ & $3(\mathrm{PGE} / \mathrm{A})$ & 1 (PMC/F) & $2(\mathrm{PAG} / \mathrm{Y})$ & $3(\mathrm{PGE} / \mathrm{A})$ \\
\hline Runtime $(\mathrm{s})^{*}$ & $1570.6(1134.1)$ & $2318.6(1192.3)$ & $3319.8(737.28)$ & $2256.2(830.78)$ & 2398.6(1202.95) & $3518.6(216.22)$ \\
\hline Load Factor* & $0.72(0.04)$ & $0.69(0.03)$ & $0.64(0.17)$ & $0.72(0.06)$ & $0.66(0.04)$ & $0.64(0.14)$ \\
\hline Irregularity Ratio* & $0(0)$ & $0(0)$ & $0(0)$ & $0.01(0.02)$ & $0.03(0.03)$ & $0.03(0.02)$ \\
\hline ASR1: Stability* & $1(0)$ & $1(0)$ & $1(0)$ & $1(0)$ & $1(0)$ & $1(0)$ \\
\hline ASR2: Max. Weight* & $1(0)$ & $1(0)$ & $1(0)$ & $1(0)$ & $1(0)$ & $1(0)$ \\
\hline ASR3: Floor Load* & $1(0)$ & $1(0)$ & $1(0)$ & $1(0)$ & $1(0)$ & $1(0)$ \\
\hline ASR4a: Balance, X-Axis* & $0.93(0.07)$ & $1(0.01)$ & $1(0)$ & $0.92(0.08)$ & $0.96(0.05)$ & $0.98(0.02)$ \\
\hline ASR4c: Balance, Y-Axis* & $0.54(0.07)$ & $0.61(0.04)$ & $0.61(0.04)$ & $0.55(0.06)$ & $0.59(0.06)$ & $0.59(0.06)$ \\
\hline ASR4b: Balance, Z-Axis* & $0.99(0.01)$ & $0.96(0.03)$ & $0.99(0.01)$ & $0.99(0.02)$ & $0.97(0.03)$ & $0.98(0.03)$ \\
\hline ASR5a: Incompatibility, Min. Distance* & $1(0)$ & $1(0)$ & $1(0)$ & $1(0)$ & $1(0)$ & $1(0)$ \\
\hline ASR5b: Incompatibility, Horiz. Separation* & $1(0)$ & $1(0)$ & $1(0)$ & $1(0)$ & $1(0)$ & $1(0)$ \\
\hline ASR5c: Incompatibility, Vert. Separation* & $1(0)$ & $1(0)$ & $1(0)$ & $1(0)$ & $1(0)$ & $1(0)$ \\
\hline ASR6: Contour* & $1(0)$ & $1(0)$ & $1(0)$ & $1(0)$ & $1(0)$ & $1(0)$ \\
\hline ACP1: Load Priority ${ }^{*}$ & $0.36(0.11)$ & $0.39(0.13)$ & $0.88(0.18)$ & $0.33(0.04)$ & $0.32(0.17)$ & $0.84(0.09)$ \\
\hline ACP2: Stacking* & $1(0)$ & $1(0)$ & $1(0.01)$ & $0.99(0.02)$ & $0.99(0.02)$ & $0.99(0.01)$ \\
\hline ACP3: Item Grouping* & $0.6(0.12)$ & $0.77(0.14)$ & $0.88(0.17)$ & $0.73(0.12)$ & $0.69(0.19)$ & $0.87(0.09)$ \\
\hline
\end{tabular}

The backend was implemented in Java, and the experiments were executed on common consumer hardware. The hardware specifications include the use of an AMD Ryzen ThreadRipper 2950X with 3.5 $\mathrm{GHz}, 16$ cores, 32 threads, and $32 \mathrm{MB}$ cache capacity and Ubuntu 18.04 as the operating system. The hardware had access to a total of 64 GB DDR4 2133 MHz/PC4-17000 CL13 RAM. The population size of the GA was set to 8,000 , and the algorithm was terminated after 300 iterations or one hour, whichever came first. The population size set was based on empirical values from previous test runs to achieve appropriate runtimes for the solution calculations. The time window was restricted based on the initial assumption that comparable restricted time windows exist within a cargo hub.

Each scenario-pallet/contour combination was tested on five flight segments. To make the evaluation visible, we captured the measured values for total runtime, load factor, and irregularity ratio. The load factor is one of the most important metrics as it measures the amount of volume used for a loaded pallet. The irregularity ratio reflects the ability of the solution design to handle the complexity of loading irregular shapes and is defined as the percentage of loaded items with irregular shapes compared to all loaded items on the pallet.

In addition, in Table 1, we present the measured values for a significant part of our assessment functions. These include all aviation safety-related constraints (ASR1-6) as well as the assessment functions for ASR4 and ASR5 consisting of their individual sub-assessments. For ASR4, the balancing of a pallet is rated along the $\mathrm{x}-, \mathrm{y}-$, or $\mathrm{z}$-axis. In turn, ASR5 includes positioning (minimum distance between items) and separation constraints, the latter of which is checked over a three-dimensional area (horizontal and vertical). To map economic and practical requirements for the generated palletizing layouts, the ACP1-3 constraints, which are not relevant for aviation safety, also need to be included. Further, ACP4 to ACP7 are intended to support the calculation of practicable palletizing layouts and must all be fulfilled. For this reason, the measured results are not explicitly listed here. Specific examples for the fulfillment of ACP8 and ACP9 are already covered by ASR5. ACP10 is not considered as Brandt and Nickel's dataset [5] does not currently provide information on complete delivery at the item level. The feasibility of ACP10 is proven by the very similar ACP3 constraint, which is a soft constraint and therefore has a tolerance range.

The results show that the runtime for the solution calculations is comparatively high. Conducting several batch runs is common in operational business and depends on the physical presence of the items at the cargo hub. Some IDSS calculation runs to generate palletizing layouts can be started earlier and have a longer time window of up to several hours, while later runs have to be executed almost in real time. The solution meets the requirements for the first runs, but the runtime must be significantly accelerated to satisfy real-time operations. The high runtimes can mainly be explained by the assessment of the solution's stability: 
namely, with the physical simulation, each palletizing layout is always built up piece by piece with a high degree of physical realism. While the simulation resolution can be lowered to achieve acceptable runtimes, such adjustment also lowers the accuracy of the physical calculations. Therefore, the key to optimizing runtimes is to significantly accelerate the physical simulation.

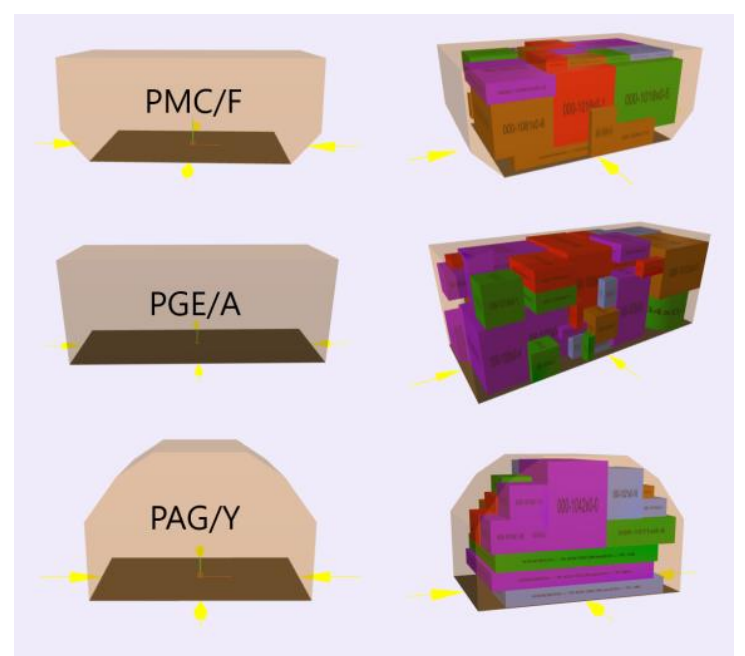

Figure 6. Visualization of pallet types, contours, and calculated palletizing layouts

The load factor is around 70\%, which is a good ratio considering that the solution fulfills all aviation safety-related constraints. As mentioned in Section 4.1., a load factor of $75 \%$ or above is aimed for in practice, but is not necessarily always useful if the emphasis is on practicable and loadable palletizing layouts. In addition, a relatively large number of heterogeneous irregular cargo items are selected for loading, if available. This reflects our efforts to explicitly address the complexity of loading heterogeneous irregular items.

The consideration of aviation safety-related constraints is almost completely fulfilled. Specifically, with regard to the balance constraints, perfect balance on $\mathrm{x}$ - and $\mathrm{z}$-axes is very difficult to achieve because a minimal deviation of the center of gravity of the load device from the geometric center is already penalized by the assessment of the GA. For the y-axis, a desirable constraint on equilibrium brings the center of gravity as low as possible, closer to the bottom of the load device, which is more difficult for high layouts. The stacking constraint is also met. In practice, fragile cargo can be supported from above by palletizing additional loading equipment, such as wooden pallets, to achieve better weight distribution from above. This special case is not yet supported in the IDSS.
The measured economically relevant constraints of load priority, stacking, and item grouping have the greatest potential for improvement. Since they are soft constraints and are not relevant to aviation safety, improvements are always desirable and will be important when comparing our IDSS results with the performance of a human palletizer. All hard constraints, including non-overlap, orthogonality, item orientation, and complexity, are met by the solution design, but, as already mentioned, are not presented here.

\section{Discussion and Conclusion}

With our evaluation, we demonstrated the feasibility of generating practicable solutions while maintaining an acceptable load factor using the IDSS. Although a practical load-factor threshold has not yet been reached, the results represent solid progress. This progress is especially apparent as almost all of the constraints mentioned above were considered when generating the solution for the palletizing layouts, which increases the complexity considerably. From this point of view, acceptable load factors as well as satisfactory treatment of the complexity were achieved. Overall, we demonstrate that is possible to plan the palletizing of cargo items on a load device with a suitable time window for planning.

A significant limitation of this study is the dataset we used. Currently, there is no realistic dataset containing relevant meta-information, such as material, weight distribution, or substructure. Also, this paper focuses strongly on the system's design and backend. This means that the current task-oriented UI for the different roles must be advanced to ensure the successful and practical use of the IDSS in the future. Such advancement must occur before an evaluation with a human palletizer can be conducted to assess the solution-generation capabilities, the transformed processes, and the user experience. In addition, runtime is another challenge that will increase with the addition of even more complex item forms and possible further constraints. The current system is clearly too slow for real-time use.

Nevertheless, we conclude that our solution approach demonstrates feasibility and that the technical evaluation indicates satisfactory performance. The proof of feasibility is also valid, with restrictions, for the runtime of the solution generation and is at the same time connected with the physical simulation of the stability assessment. Further research in this field can be extended to the use of graphical processor units for the physical calculation of parallelization, which are significantly more efficient. Further approaches to improve the IDSS 
include the addition of the yet to be integrated constraints, such as complete shipment, and by enhancing and evaluating the existing constraints by analyzing the solution generation of the palletizing layouts and the layouts themselves. Furthermore, the possibilities of the GA have not yet been fully explored. The goal is to achieve greater population sizes and higher iterations in the same time window to improve the GA's solution generation by optimizing or enhancing the current algorithm. The simultaneous consideration of both areas-the algorithm and constraints - supports further development to reach a real-time system. Last but not least, practice-oriented research based on a realistic dataset is strongly recommended when such a dataset becomes available.

\section{Acknowledgement}

We would like to thank the cargo experts from Lufthansa Cargo AG for their support in conducting this project. This research was partly funded by the Innovation Growth Fund of the Lufthansa Group.

\section{References}

[1] Airbus, Global Market Forecast - Cities, Airports \& Aircraft - 2019-2038, Airbus S.A.S., Blagnac Cedex, 2019,

[2] Bischoff, E.E., Janetz, F., and Ratcliff, M.S.W., "Loading pallets with non-identical items", European Journal of Operational Research 84 (3), 1995, pp. 681-692.

[3] Bischoff, E.E. and Ratcliff, M.S.W., "Issues in the development of approaches to container loading", Omega 23 (4), 1995, pp. 377-390.

[4] Bortfeldt, A. and Wäscher, G., "Constraints in container loading-A state-of-the-art review", European Journal of Operational Research 229 (1), 2013, pp. 1-20.

[5] Brandt, F. and Nickel, S., "The air cargo load planning problem - a consolidated problem definition and literature review on related problems", European Journal of Operational Research 275 (2), 2019, pp. 399-410.

[6] BVL, Fachkräftemangel in der Logistik - Eine Umfrage der BVL, Bremen, 2017, .

[7] Cagan, J., Shimada, K., and Yin, S., "A survey of computational approaches to three-dimensional layout problems", Computer-Aided Design 34 (8), 2002, pp. $597-$ 611.

[8] Chan, F.T.S., Bhagwat, R., Kumar, N., Tiwari, M.K., and Lam, P., "Development of a decision support system for aircargo pallets loading problem: A case study", Expert Systems with Applications 31 (3), 2006, pp. 472-485.

[9] Davies, A.P. and Bischoff, E.E., "Weight distribution considerations in container loading", European Journal of Operational Research 114 (3), 1999, pp. 509-527.

[10] Egeblad, J., Garavelli, C., Lisi, S., and Pisinger, D., "Heuristics for container loading of furniture", European Journal of Operational Research 200 (3), 2010, pp. 881-892.
[11] Graver, B., Zhang, K., and Rutherford, D., CO2 emissions from commercial aviation, 2018. Working Paper, International Council on Clean Transportation, Berlin, 2019,

[12] Gregory, R.W. and Muntermann, J., "Research Note Heuristic Theorizing: Proactively Generating Design Theories", Information Systems Research 25 (3), 2014, pp. 639-653.

[13] Hodgson, T.J., "A Combined Approach to the Pallet Loading Problem”, A I I E Transactions 14 (3), 1982, pp. 175-182.

[14] Hong Ha, H.T. and Nananukul, N., “Air Cargo Loading Management System for Logistics Forwarders", ICUPTCE16, Jan. 2-3, 2016, Pattaya, Thailand, Proceedings, 2016, pp. $51-58$.

[15] IATA, Cargo Handling Manual, International Air Transport Association, Montreal-Geneva, 2018, .

[16] IATA, Air Freight Market Analysis - May 2019, International Air Transport Association, Montreal-Geneva, 2019,

[17] Kovynyov, I. and Mikut, R., "Digital technologies in airport ground operations", NETNOMICS: Economic Research and Electronic Networking 20 (1), 2019, pp. 1-30.

[18] Kramer, O., Genetic Algorithm Essentials, Springer International Publishing, Cham, 2017,

[19] Krish, S., "A practical generative design method", Computer-Aided Design 43 (1), 2011, pp. 88-100.

[20] Morrell, P.S. and Klein, T., Moving Boxes by Air, Routledge, Abingdon-New York, 2019,

[21] Paquay, C., Limbourg, S., Schyns, M., and Oliveira, J.F., "MIP-based constructive heuristics for the threedimensional Bin Packing Problem with transportation constraints", International Journal of Production Research 56 (4), 2018, pp. 1581-1592.

[22] Paquay, C., Schyns, M., and Limbourg, S., "A mixed integer programming formulation for the three-dimensional bin packing problem deriving from an air cargo application", International Transactions in Operational Research 23 (1-2), 2016, pp. 187-213.

[23] Peffers, K., Tuunanen, T., Rothenberger, M.A., and Chatterjee, S., "A Design Science Research Methodology for Information Systems Research", Journal of Management Information Systems 24 (3), 2007, pp. 45-77.

[24] Pollaris, H., Braekers, K., Caris, A., Janssens, G.K., and Limbourg, S., "Vehicle routing problems with loading constraints: state-of-the-art and future directions", OR Spectrum 37 (2), 2015, pp. 297-330.

[25] Singh, V. and Gu, N., "Towards an integrated generative design framework”, Design Studies 33 (2), 2012, pp. 185-207.

[26] Wäscher, G., Haußner, H., and Schumann, H., "An improved typology of cutting and packing problems", European Journal of Operational Research 183 (3), 2007, pp. 1109-1130.

[27] Zhao, X., Bennell, J.A., Bektaş, T., and Dowsland, K., "A comparative review of 3D container loading algorithms", International Transactions in Operational Research 23 (1-2), 2016, pp. 287-320. 